\section{Lung cancer signatures in blood}

\author{
By Kai-Jye Lou, Staff Writer
}

U.S. researchers have profiled and compared the proteomes of multiple mouse models to identify plasma protein signatures that could aid the discovery of lung cancer biomarkers. ${ }^{1}$ Key questions include whether the blood biomarkers could be used for initial screening of patients or whether they will be better suited as a noninvasive follow-up test to complement imaging-based diagnostic approaches.

The 5-year survival rate for U.S. lung cancer patients with localized disease is about $53 \%$ compared with $24 \%$ and $4 \%$ for those diagnosed with regional and distant disease, respectively. ${ }^{2}$ Despite the clear importance of catching the disease as early as possible, there are no validated blood-based biomarkers that are sufficiently sensitive and specific to detect early stage lung cancer. ${ }^{3}$

"We previously did plasma analysis on mouse models of other cancers, and we were pretty impressed with the relevance of
"The work shows that tumors do yield blood-based signatures early during development. It also shows that it would be feasible to develop blood tests to detect common cancer types at an early stage."

- Samir Hanash,

Fred Hutchinson Cancer Research Center and SCLC-associated signatures that best distinguished each disease subtype and for which assays were available and used them to screen human samples.

In plasma samples from newly diagnosed lung cancer patients and matched healthy controls, immunoassays showed that the signature subsets could determine whether the individual had the disease. In serum samples taken up to 11 months prior to a patient being diagnosed with NSCLC and samples from control subjects, the immunoassays picked ut those from patients that were eventually diagnosed with the disease.

The prediagnosis samples were taken from a repository for a clinical trial that evaluated vitamin A supplementation for the prevention of lung

Results were published in Cancer Cell.

"The work shows that tumors do yield blood-based signatures early during development," said Hanash, who is corresponding author on the paper. "It also shows that it would be feasible to develop blood tests to detect common cancer types at an early stage."

"The results are encouraging since they not only verify known oncogenic pathways in specific histological lung cancer mouse models using a high throughput proteomic approach but also demonstrate the potential of specific proteins to diagnose cancer in human samples," said Duncan Whitney, VP of R\&D at Allegro Diagnostics Inc.

"One of the primary benefits of this approach appears to be an ability to profile a broad range of serum proteins using high-sensitivity mass spectrometry," Whitney told SciBX. "This approach may be useful in future studies to tailor tests to other specific indications, such as the findings to human cancer," said Samir Hanash, program head of molecular diagnostics at the Fred Hutchinson Cancer Research Center. "We therefore pursued an intensive search for lung cancer markers based on mouse models of lung cancer."

The group used quantitative mass spectrometry to profile the plasma proteomes of mice with lung cancer, other types of solid tumors or inflammation. The researchers then mined the resulting proteomic dataset for protein signatures that could reflect lung tumor biology.

From their analyses, the team identified plasma protein signatures for small cell lung cancer (SCLC) and lung adenocarcinoma, which is the most common subtype of non-small cell lung cancer (NSCLC). Each signature is a panel of proteins that are differentially expressed in lung cancer relative to the other disease models.

The group also found that the signatures could help assess treatment response. In a mouse model of epidermal growth factor receptor (EGFR)mutant lung adenocarcinoma, 91 of 164 proteins with levels that were increased or decreased moved back toward baseline after the animals were treated with Tarceva erlotinib.

Tarceva is marketed by Astellas Pharma Inc., Chugai Pharmaceutical Co. Ltd. and Roche for NSCLC.

The researchers then selected subsets of proteins from the NSCLC- therapeutic response indicators for a variety of drugs."

Allegro is developing its BronchoGen genomic test for use in combination with standard bronchoscopy for the early diagnosis of lung cancer. The test, which the company plans to launch next year, analyzes the expression of a panel of genes in bronchial epithelial cells obtained from a bronchoscopy.

"This very rich proteomic dataset is now available for further data mining, hypothesis generation and validation of candidates," said Pierre Massion, an associate professor of medicine and cancer biology at the Vanderbilt-Ingram Cancer Center. "What I found particularly impressive is that the group tested the candidate markers in a cohort of individuals at high risk for lung cancer and showed that these markers were associated with those that were eventually diagnosed with the disease."

"As experience is gained, we envisage that a blood test may become the initial screening modality" for lung cancer, Hanash told SciBX.

Another possibility is to use a blood test as an adjunct to CT scans.

Lung cancer is typically first detected from a standard chest X-ray or CT scan. In June, data from the National Lung Screening Trial showed that screening with CT scans decreased lung cancer mortality by $20 \%$ relative to screening with chest X-rays. ${ }^{4}$ However, CT scans can pick up 


\section{ANALYSIS}

indeterminate nodules in the lung that require additional follow-up and have a high false positive rate.

Dimo Dietrich, head of technology research at Epigenomics AG, said these problems are the main reasons CT scans have not yet been

"A defined strategy, possibly including biomarkers, for assisting in the management of indeterminate lung nodules would be an appropriate consideration before the implementation of CT as a standard method for lung cancer screening."

-Dimo Dietrich, Epigenomics AG recommended for routine lung cancer screening.

"A defined strategy, possibly including biomarkers, for assisting in the management of indeterminate lung nodules would be an appropriate consideration before the implementation of CT as a standard method for lung cancer screening," he told SciBX.

Dietrich added that for screening, the key for any useful lung cancer biomarker will be the ability to pick out patients who still have localized disease, given the poor prognosis for disease that has advanced beyond this stage.

Epigenomics markets its Epi proLung BL Reflux assay in Europe to help confirm malignant disease in patients suspected of having lung cancer. The in vitro diagnostic detects methylated DNA of the short stature homeobox 2 (SHOX2; SHOT) gene in bronchial lavage fluid and has CE mark approval.

Methylated SHOX2 is a biomarker that can help differentiate between malignant and normal lung tissues. ${ }^{5}$

The BronchoGen genomic test and Epi proLung BL Reflux assay are not blood tests.

\section{The validation gap}

Hanash said his group is now pursuing the necessary validation studies and developing assays for additional protein markers found in the plasma signatures.
"The work is a discovery effort with some early validation, and while the results are encouraging, they are still far from clinical applications," Massion told SciBX. "It clearly needs validation in larger cohorts, and the validation work needs to be emphasized and encouraged just as much as the nice discovery work carried out in the current study. The candidate biomarkers or signatures need to be tested in the clinical context within different cohorts and be integrated with other available predictive tools."

"Follow-on clinical studies using prospectively collected human sera samples would be helpful to demonstrate the suitability of this approach in a larger sample set," added Whitney. "It would also be useful to confirm the ability of serum proteins to detect early stage lung cancers in order to begin to make inroads to reducing mortality in lung cancers."

The Office of Industry Relations and Technology Transfer at the Fred Hutchinson Cancer Research Center declined to disclose the patent and licensing status of the work.

Lou, K.-J. SciBX 4(39); doi:10.1038/scibx.2011.1083

Published online Oct. 6, 2011

\section{REFERENCES}

1. Taguchi, A. et al. Cancer Cell; published online Sept. 12, 2011; doi:10.1016/j.ccr.2011.08.007

Contact: Samir M. Hanash, Fred Hutchinson Cancer Research Center, Seattle, Wash.

e-mail: shanash@fhcrc.org

2. Jemal, A. et al. CA Cancer J. Clin. 60, 277-300 (2010)

3. Hanash, S.M. et al. Nat. Rev. Clin. Oncol. 8, 142-150 (2011)

4. National Lung Screening Trial Research Team N. Engl. J. Med. 365, 395-409 (2011)

5. Schmidt, B. et al. BMC Cancer 10, 600 (2010)

COMPANIES AND INSTITUTIONS MENTIONED

Allegro Diagnostics Inc., Maynard, Mass.

Astellas Pharma Inc. (Tokyo:4503), Tokyo, Japan

Chugai Pharmaceutical Co. Ltd. (Tokyo:4519), Tokyo, Japan

Epigenomics AG (Xetra:ECX), Berlin, Germany

Fred Hutchinson Cancer Research Center, Seattle, Wash.

Roche (SIX:ROG; OTCQX:RHHBY), Basel, Switzerland

Vanderbilt-Ingram Cancer Center, Nashville, Tenn. 